\title{
Assessment of Efficacy of Virtual Reality Distraction in Reducing Pain Perception and Anxiety in Children Aged 6-10 Years: A Behavioral Interventional Study
}

\author{
Dhanu G Rao ${ }^{1}$, Raghavendra Havale ${ }^{2}$, Manasa Nagaraj ${ }^{3}$, Namira M Karobari ${ }^{4}$, Athira M Latha ${ }^{5}$, Namratha Tharay ${ }^{6}$, \\ Santhebachalli P Shrutha7
}

\begin{abstract}
Aim: The aim of the present study was to assess the effectiveness of virtual reality distraction on pain perception and state anxiety levels undergoing restorative treatment in children.

Materials and methods: This was an interventional study with 30 children of age 6-10 years came to the Department of Pedodontics and Preventive Dentistry. The intervention was distraction with virtual reality eyeglasses and the parameters considered includes pain perception analyzed subjectively by Wong Baker FACES pain rating scale and objectively by FLACC scale; anxiety was analyzed physiologically by measuring pulse rate and oxygen saturation levels using pulse oximeter. The parameters were recorded before the treatment, i.e., baseline, during and as well as after the restorative treatment procedure. The values noted were tabulated and subjected to appropriate statistical analysis with $p$ value set at 0.05 .

Results: The study displayed a very high statistical significance in reduction of pain perception and anxiety levels in all the comparisons made at three time periods, i.e., baseline, during and after treatment procedure $(p<0.0001)$.

Conclusion: Virtual reality distraction can be considered as a budding distraction tool in the arena of behavior management that helps adapt the child to dental environment and able to deliver qualitative dental care.

Clinical significance: Managing an anxious child is one of the challenging tasks for a pediatric dentist in the day-to-day life. As the world progresses with newer interventions, virtual reality distraction is one among them that has the ability to reduce pain perception and anxiety in children with a positive approach.

Keywords: Behavior, Children, Dental anxiety, Interventional study, Pain, Virtual reality eyeglasses.

International Journal of Clinical Pediatric Dentistry (2019): 10.5005/jp-journals-10005-1694
\end{abstract}

\section{INTRODUCTION}

The term pain and anxiety are the most annoying feelings and emotional experiences, that are associated with real or possible traumas to tissues. ${ }^{1}$ Pain is a subjective one and its perception depends on several factors like physiological, psychological, social, culture and to some extent on genetics. The perception of pain increases when the child is consciously attentive in that particular environment and is also related to the appalling thinking about pain, anxiety and fear associated with it, respectively. ${ }^{2}$

Both pain perception and dental anxiety in children leads to the avoidance of dental treatment which in turn results in deterioration of oral health, leading to more problem-oriented visiting, which serves to maintain or exacerbate the person's level of dental anxiety. In children, if treatment was given without alleviation of anxiety, not only hampers the quality of dental care, it also creates a undesirable impact of their judgment on dentistry in future. Henceforth, it is of great importance that the dental health professionals should be able to detect the children with dental anxiety and apply appropriate behavior management techniques at the earliest age possible. ${ }^{3,4}$

Management strategies have been projected to minimize pain and discomfort during dental treatment in children. They are mainly divided into two broad categories. The first module consists of nonpharmacological behavioral techniques like the tell-show-do technique, distraction, modeling and hypnotism. The second category consists of pharmacologic techniques. ${ }^{5,6}$ Distraction is
${ }^{1-7}$ Department of Pedodontics and Preventive Dentistry, AME's Dental College and Hospital, Raichur, Karnataka, India

Corresponding Author: Dhanu G Rao, Department of Pedodontics and Preventive Dentistry, AME's Dental College and Hospital, Raichur, Karnataka, India, Phone: +91 9845031755, e-mail: drdhanu74@yahoo. co.in

How to cite this article: Rao DG, Havale R, Nagaraj M, et al. Assessment of Efficacy of Virtual Reality Distraction in Reducing Pain Perception and Anxiety in Children Aged 6-10 Years: A Behavioral Interventional Study. Int J Clin Pediatr Dent 2019;12(6):510-513.

Source of support: Nil

Conflict of interest: None

the technique of diverting the children attention from what may be professed as an unpleasant procedure and was one of the most commonly used and effective behavior guidance technique. The newer method of distraction that is gaining popularity in the present is virtual reality distraction (VRD).

Virtual reality (VR) refers to "a human-computer interface that allows the user to interact dynamically with the virtual world, which is essentially a computer-generated environment." The application of virtual reality as a distraction technique could perhaps be superior to traditional distraction techniques because "it offers more immersive images via the occlusive headsets that project 
the images right in front of the eyes of the user." Depending on the model of VR device used, it may block out the real-world by hindering the visual, auditory, or both the stimuli. ${ }^{7}$

So, the aim of present study was to assess the pain perception and anxiety of children by using virtual reality distraction. The hypothesis will be null hypothesis, i.e., neither or nor virtual reality distraction is related to anxiety and pain perception.

\section{Materials and Methods}

\section{Source of the Data}

After obtaining Institutional Ethical Clearance as per the code of ethics of the World Medical Association and declaration of Helsinki, parents' consent form, children aged 6-10 years were selected based on the inclusion criteria from the OPD of Department of Pedodontics and Preventive Dentistry, AME's Dental College and Hospital, Raichur, Karnataka from September 2018 to October 2018.

\section{Study Design}

The present study was an interventional clinical study with parallel design and virtual reality distraction being the intervention.

\section{Sample Size Determination}

Based on the previous pilot study, taking $80 \%$ power and $5 \%$ margin of error $(1-\beta)$ and a total of 30 children were considered as appropriate sample size to fulfill the purpose of the study.

\section{Methods}

Children with Frankl's behavior 3 and 4, who are free from any psychological as well as systemic illness, without any anxiety disorders and with a requisite for restorative dental procedure, were included in the study. The parameters assessed were pain perception and anxiety before, during and after the procedure. Pain was measured in two ways by using self-reported Wong Baker faces pain scale (WBFPS) and objectively by FLACC scale. Anxiety was assessed physiologically by using pulse oximeter (Nellcor COVIDIEN $\mathrm{SPO}_{2}$ sensor compatible planet 50n LT model) in which pulse rate and oxygen saturation was monitored. Virtual reality distraction (VRD) eyeglasses (ANTVR Phone Glass T2, Model PA15LF53A) with a choice of three cartoons that is left for their choice was given to the child before the start of the restorative procedure and they were removed only after the completion of the procedure, if any inconvenience was encountered by the child during the procedure they were advised to inform by using signalling system. Prior to the start of the procedure the child was made adaptive by elucidation about the eyeglasses. One examiner who was accustomed with the scales recorded all the parameters. The data was analyzed using SPSS version 21.0 and subjected to statistical analysis.

\section{Statistical Analysis}

Descriptive and inferential statistics were applied wherever required. The results were expressed in mean and standard deviation, Wilcoxon signed rank test and paired " $t$ " test was used in the present study to evaluate the parameters accordingly. The statistical significance was set at 0.05 .

\section{Results \\ Demographic Data}

Among 30 children recruited into the study, 18 (60\%) were boys and 12 (40\%) were girls. The mean age of the children was $7.77 \pm$ 1.88 (Table 1).

\section{Pain Perception \\ Subjective Assessment of Pain}

Pain perception that was rated by Wong Baker FACES pain rating scale was compared at baseline, during and after restorative procedure, very high statistical significance was found $(p<0.0001)$ (Fig. 1). The mean scores were $8.07 \pm 2.20$ and $3.13 \pm 1.25$ when baseline to during treatment was compared and to baseline and after treatment, mean score is $8.07 \pm 2.20$ and $1.07 \pm 1.26$, respectively. The mean scores during treatment and after treatment were $3.13 \pm 1.25$ and $1.07 \pm 1.26$, respectively. This assessment stipulates that pain perception in children was reduced by using visual reality distraction.

\section{Objective Assessment of Pain}

When pain was assessed objectively, the mean score of children from baseline to during treatment is $6.77 \pm 1.79$ and $2.57 \pm 1.01$; from baseline to after treatment it is $6.77 \pm 1.79$ and $0.24 \pm 0.74$,

Table 1: Demographic data

\begin{tabular}{ll}
\hline Boys & $18(60 \%)$ \\
Girls & $12(40 \%)$ \\
\hline
\end{tabular}

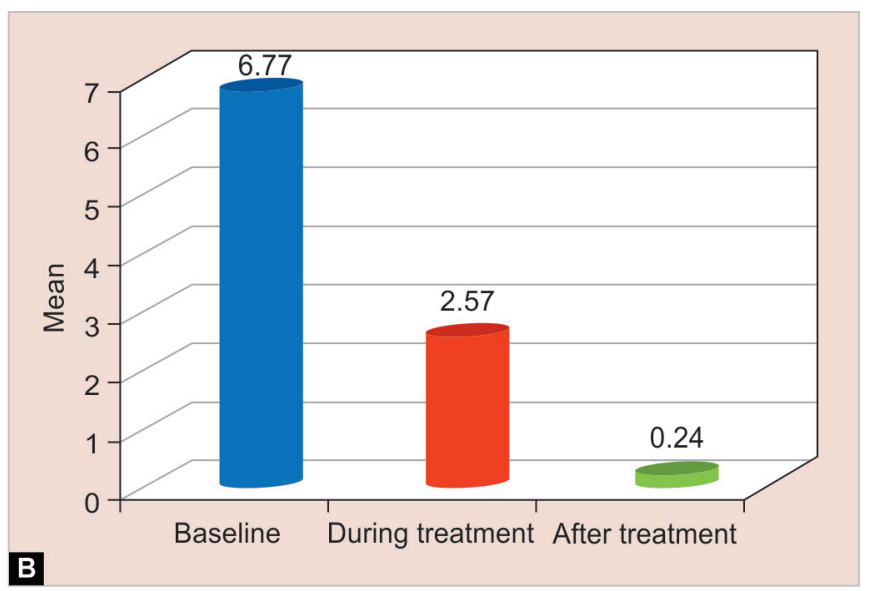

B

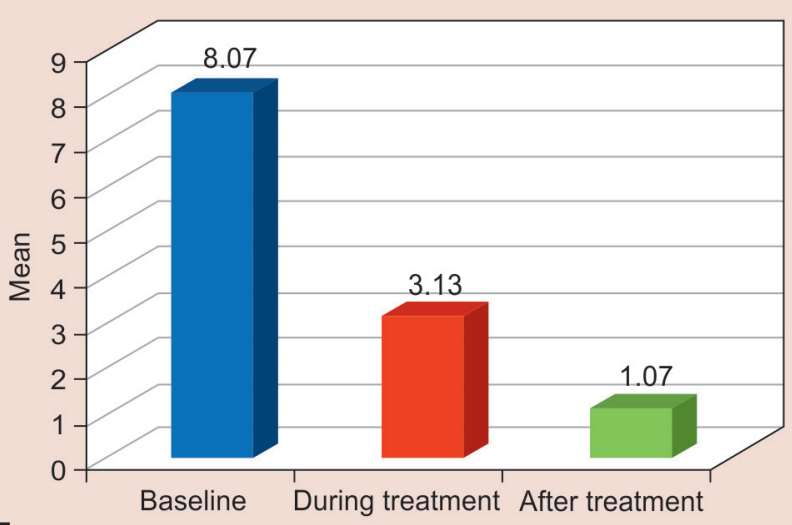

A

Figs 1 A and B: Assessment of pain perception scores rated by (A) Wong Baker FACES pain rating scale; (B) FLACC scale 
respectively, during treatment and after treatment it is $2.57 \pm 1.01$ and $0.24 \pm 0.74$, respectively (Table 2 ). Comparisons between them was found to be very high statistically significant $(p<0.0001)$ (Fig. 1).

\section{Anxiety}

\section{Assessment of Pulse Rate}

On comparing pulse rate of study participants at different time intervals there is a gradual decrease in pulse rate observed from baseline to during treatment and after treatment which was highly statistical significance $(p<0.0001)$, mean scores being $104.97 \pm 9.74$ and $93.53 \pm 8.60$, respectively (Fig. 2). Pulse rate when compared during and after treatment was increased that was high statistically significant, mean score is $93.53 \pm 8.60$ and $95.97 \pm 7.62(p<0.001)$ (Table 3).

\section{Assessment of Oxygen Saturation}

When oxygen saturation was analyzed, there was an increase from baseline to during treatment and after treatment with the high statistical significance ( $p<0.0001^{*}$ ) (Fig. 2). The levels when

Table 2: Comparison of Wong Baker FACES pain rating scale and FLACC scale

\begin{tabular}{|c|c|c|c|}
\hline & $N$ & Mean $\pm(S D)$ & $\begin{array}{l}\text { Wilcoxon signed } \\
\text { rank test } p \text { value }\end{array}$ \\
\hline \multicolumn{4}{|c|}{ Wong-Baker FACES pain rating scale } \\
\hline Baseline & 30 & $8.07 \pm 2.20$ & $0.0001^{*}$ \\
\hline During treatment & 30 & $3.13 \pm 1.25$ & \\
\hline Baseline & 30 & $8.07 \pm 2.20$ & $0.0001^{*}$ \\
\hline After treatment & 30 & $1.07 \pm 1.26$ & \\
\hline During treatment & 30 & $3.13 \pm 1.25$ & $0.0001^{*}$ \\
\hline After treatment & 30 & $1.07 \pm 1.26$ & \\
\hline \multicolumn{4}{|l|}{ FLACC scale score } \\
\hline Baseline & 30 & $6.77 \pm 1.79$ & $0.0001^{*}$ \\
\hline During treatment & 30 & $2.57 \pm 1.01$ & \\
\hline Baseline & 30 & $6.77 \pm 1.79$ & $0.0001^{*}$ \\
\hline After treatment & 30 & $0.24 \pm 0.74$ & \\
\hline During treatment & 30 & $2.57 \pm 1.01$ & $0.0001^{*}$ \\
\hline After treatment & 30 & $0.24 \pm 0.74$ & \\
\hline
\end{tabular}

*Very high statistical significance. $N$, number; SD, standard deviation compared during and after treatment, the mean scores were almost same, i.e., $99.8 \pm 0.41$ and $99.87 \pm 0.35$, respectively but they are statistically insignificant $(p=0.161)$ (Table 3$)$.

\section{Discussion}

As the anxiety was the most common problem encountered in the pediatric dentistry, that illustrates its effects on the child, parent as well as the dentist in coping and rendering qualitative dental treatment. The present study was carried out mainly to assess the pain perception and anxiety while the VRD was applied. The validity and reliability of the scales (WBFPS and FLACC) used in the present study was previously evaluated by many studies. ${ }^{8-10}$

The result of the present study was in accordance to Shetty et al., ${ }^{11}$ Chaturvedi et al., ${ }^{12}$ Asl Aminabadi et al., ${ }^{8}$ Wiederhold et al. ${ }^{13}$ and Sullivan et al., ${ }^{14}$ where both the pain perception and anxiety were reduced. As both the pain perception and anxiety increase if the child focusses on the procedure or sight of instruments in dentistry; the child was taken into a different environment by applying VRD which is devoid of the operator's field and its sounds.

Table 3: Comparison of pulse rate and oxygen saturation levels

\begin{tabular}{lrrl}
\hline & & & \multicolumn{1}{c}{$\begin{array}{l}\text { Paired } \\
\text { t test } p \text { value }\end{array}$} \\
\hline Pulse rate & & & \\
Baseline & 30 & $104.97 \pm 9.74$ & $0.0001^{*}$ \\
During treatment & 30 & $93.53 \pm 8.60$ & \\
Baseline & 30 & $104.97 \pm 9.74$ & $0.0001^{*}$ \\
After treatment & 30 & $95.97 \pm 7.62$ & \\
During treatment & 30 & $93.53 \pm 8.60$ & $0.001^{* *}$ \\
After treatment & 30 & $95.97 \pm 7.62$ & \\
Oxygen saturation level & & & \\
Baseline & 30 & $98.2 \pm 0.71$ & $0.001^{* *}$ \\
During treatment & 30 & $99.8 \pm 0.41$ & \\
Baseline & 30 & $98.2 \pm 0.70$ & $0.001^{* *}$ \\
After treatment & 30 & $99.87 \pm 0.35$ & \\
During treatment & 30 & $99.8 \pm 0.41$ & 0.161 \\
After treatment & 30 & $99.87 \pm 0.35$ & \\
\hline
\end{tabular}

*Very high statistical significance; ${ }^{* * H i g h}$ statistical siginificance. $N$ number; SD, standard deviation

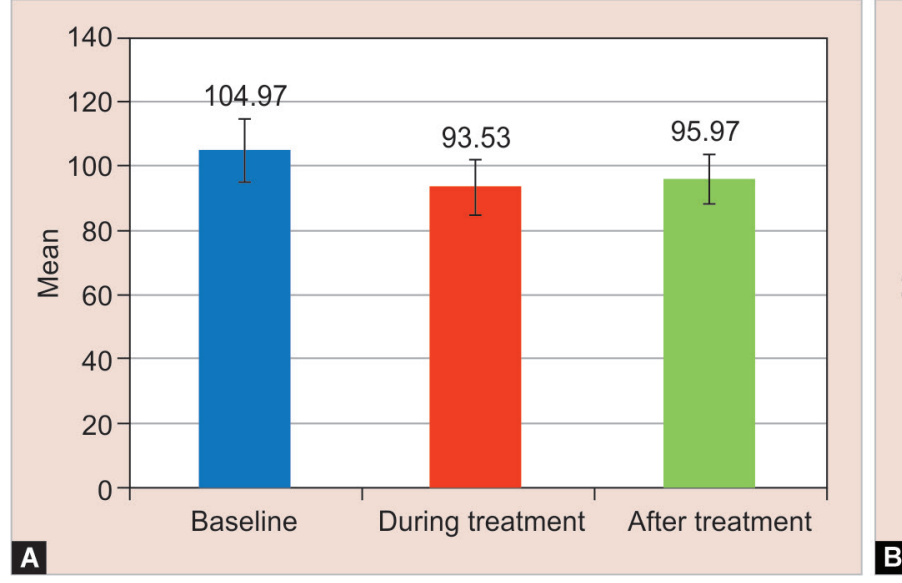

Fig. 2: Assessment of anxiety through (A) Pulse rate; (B) Oxygen saturation

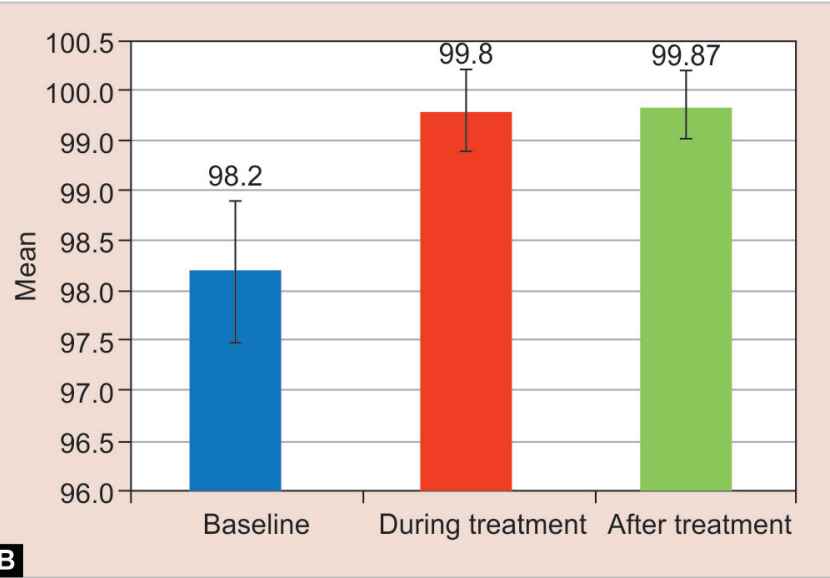


The effect of VRD can be carried after the treatment; this can be explained by comparing the oxygen saturation levels during and after the treatment but it was not found to be statistically significant. In addition, pulse rate during the procedure was illustrated to be less, which agrees that the child was in relaxed state during the procedure which is advantageous to the pediatric dentist.

The working principle of VRD is that it does not interrupt the pain signals but acts both directly and indirectly on pain perception and signalling. ${ }^{15}$ Several studies has evaluated the efficacy of VRD in the fields of medicine and dentistry and showed virtuous results. A study by Hoffman et al., compared VRD with opioids and found that virtual reality and opioid analgesics had same equivalent results in terms of pain reduction, and it was found that the combination of opioids with virtual reality ensued in significant reduction in pain signals. ${ }^{16}$ Therefore, VRD has the potential to reduce pain perception.

Furthermore, the other advantages of VRD includes its ease of usage, more acceptance, safety, economic less complexity in understanding the procedure whereas the disadvantage of VRD is communication with the child when it is in use. 8,17

In addition to this, VRD has the potential to reduce the memories during the procedure. ${ }^{18}$ So any unpleasant stimulus during the procedure does not have any effect on the future appointment. Furthermore, VRD helps to instill a positive attitude towards the dentist and dental treatments that can be carried out through adolescence into adulthood.

According to recent systematic reviews, evidence is very low that states distraction techniques to be effective in reducing fear and anxiety during dental treatment which signifies further interventional studies should be conducted. ${ }^{19}$ Furthermore, the limitations of the present study include, it would be better if different treatment procedures were evaluated separately, if both the genders are recruited equally and trait anxiety was not evaluated. One of the interesting features of VRD is that its effect on behavior modification can be carried for long-term. ${ }^{18}$ So, it throws a challenge for researchers for further long-term follow-up studies based on VRD.

\section{Conclusion}

VRD that utilizes the new VR can be considered as an effective means of behavior guidance technique that reduces the anxiety and pain perception in children.

\section{References}

1. Mathew PJ, Mathew JL. Assessment and management of pain in infants. Postgard Med J 2003;79(934):438-443. DOI: 10.1136/ pmj.79.934.438.

2. Lamarca GA, Vettore MV, Monteiro da Silva AM. The influence of stress and anxiety on the expectation, perception and memory of dental pain in schoolchildren. Dent J (Basel) 2018;6(4):E60. DOI: 10.3390/ dj6040060.

3. Holmes RD, Girdler NM. A study to assess the validity of clinical judgement in determining paediatric dental anxiety and related outcomes of management. Int J Pediatr Dent 2005;15(3):169-176. DOI: 10.1111/j.1365-263X.2005.00633.x.

4. Klaassen MA, Veerkamp JSJ, Aartman IHA, et al. Stressful situations for toddlers: indications for dental anxiety? ASDC J Dent Child 2002;69(3):306-309.

5. Navit S, Johri N, Khan SA, et al. Effectiveness and comparison of various audio distraction aids in management of anxious dental paediatric patients. J Clin Diagn Res 2015;9(12):ZC05-ZC09. DOI: 10.7860/JCDR/2015/15564.6910.

6. Singh D, Samadi F, Jaiswal JN, et al. Stress reduction through audio distraction in anxious pediatric dental patients: an adjunctive clinical study. Int J Clin Pediatr Dent 2014;7(3):149-152. DOI: 10.5005/ jp-journals-10005-1254.

7. Prabhaker AR, Marwah N, Raju OS. A comparison between audio and audiovisual distraction techniques in managing anxious pediatric dental patients. J Indian Soc Pedod Prev Dent 2007;25(4):177-182. DOI: 10.4103/0970-4388.37014.

8. Asl Aminabadi N, Erfanparast L, Sohrabi A, et al. The impact of virtual reality distraction on pain and anxiety during dental treatment in 4-6 year-old children: a randomized controlled clinical trial. J Dent Res Dent Clin Dent Prospects 2012;6(4):117-124. DOI: 10.5681/ joddd.2012.025.

9. Mitrakul K, Asvanund Y, Arunakul M, et al. Effect of audiovisual eyeglasses during dental treatment in 5-8 year-old children. Eur J Paediatr Dent 2015;16(3):239-245.

10. Nilson S, Finnstrom B, Kokinsky E, et al. The use of virtual reality for needle related procedural pain and distress in children and adolescents in a pediatric oncology unit. Eur J Oncol Nurs 2009;13(2):102-109. DOI: 10.1016/j.ejon.2009.01.003.

11. Shetty V, Suresh LR, Hegde AM. Effect of virtual reality distraction on pain and anxiety during dental treatment in 5 to 8 year old children. J Clin Pediatr Dent 2019;43(2):97-102. DOI: 10.17796/1053-462543.2.5.

12. Chaturvedi $\mathrm{S}$, Walimbe $\mathrm{H}$, Karekar $\mathrm{P}$, et al. Comparative evaluation of anxiety level during the conventional dental procedures with and without audiovisual distraction eyeglasses in pediatric dental patients. J Int Oral Health 2016;8(11):1016.

13. Wiederhold MD, Gao K, Wiederhold BK. Clinical use of virtual reality distraction system to reduce anxiety and pain in dental procedures. Cyberpsychol Behav Soc Netw 2014;17(6):359-365. DOI: 10.1089/ cyber.2014.0203.

14. Sullivan C, Schneider PE, Musselman RJ, et al. The effect of virtual reality during dental treatment on child anxiety and behavior. ASDC J Dent Child 2000;67:193-196, 160-161.

15. Arane K, Behboudi A, Goldman RD. Virtual reality for pain and anxiety management in children. Can Fam Physician 2017;63(12):932-934.

16. Hoffman HG, Garcia-Palacios A, Patterson DR, et al. The effectiveness of virtual reality for dental pain control. Cyberpsychol Behav 2001;4(4):527-535. DOI: 10.1089/109493101750527088.

17. Niharika P, Reddy NV, Srujana P, et al. Effects of distraction using virtual reality technology on pain perception and anxiety levels in children during pulp therapy of primary molars. J Indian Soc Pedod Prev Dent 2018;36(4):364-369. DOI: 10.4103/JISPPD.JISPPD_1158_17.

18. Tanja-Dijkstra K, Pahl S, White MP, et al. Can virtual nature improve patient experiences and memories of dental treatment? A study protocol for a randomized controlled trial. Trials 2014;15:90. DOI: 10.1186/1745-6215-15-90.

19. Liu Y, Gu Z, Wang Y, et al. Effect of audiovisual distraction on the management of dental anxiety in children: a systematic review. Int J Paediatr Dent 2019;29(1):14-21. DOI: 10.1111/ipd.12430. 